\title{
Line broadening in the Si I, Si II, Si III, and Si IV spectra in the helium plasma
}

\author{
S. Bukvić, S. Djeniže, and A. Srećković
}

Faculty of Physics, University of Belgrade, Studentski trg 14, 11000 Belgrade, Serbia

e-mail: ebukvic@ff.bg.ac.rs

Received 12 March 2009 / Accepted 10 September 2009

\section{ABSTRACT}

\begin{abstract}
Context. The neutral and ionized silicon spectral line shapes have been investigated in the laboratory helium plasma at electron densities ranging between $3.7 \times 10^{22} \mathrm{~m}^{-3}$ and $1.1 \times 10^{23} \mathrm{~m}^{-3}$ and electron temperatures between $12500 \mathrm{~K}$ and $19000 \mathrm{~K}$, both interesting for astrophysics.

Aims. The aim of this work is to present experimental Stark FWHM (full-width at half of the maximum line intensity, $W$ ) for number of spectral lines from neutral (Si I), singly (Si II), doubly (Si III), and triply (Si IV) ionized silicon spectra emitted by the pulsed helium discharge, which is optically thin at the wavelengths of the investigated ionic silicon lines. A specific method for estimating self-absorbtion is presented in detail. For investigated Si I spectral lines, applying the proposed method, an optical depth of less than 0.38 is found. Appropriate corrections of the Si I Stark widths were made. The Stark widths of different ionic species, presented in this paper, are measured for the first time in the essentially same laboratory plasma.

Methods. The silicon atoms were evaporated from the walls of the specially designed pyrex discharge tube in the pulsed helium discharge at a pressure of $665 \mathrm{~Pa}$ in a flowing regime. The Si I, Si II, Si III, and Si IV spectral line profiles were recorded using the McPherson model 209 spectrograph and the Andor ICCD camera as the detection system.

Results. The Stark FWHMs of 13 Si I, 15 Si II, 28 Si III, and 9 Si IV spectral lines were measured in the wavelength interval between $206 \mathrm{~nm}$ and $640 \mathrm{~nm}$. Five Si I, four Si II, eleven Si III, and one Si IV $W$ values from the above set not had measured or calculated. Our $W$ values are compared with the existing theoretical and experimental data.

Conclusions. At the mentioned plasma parameters tolerable agreement was found (within the accuracy of the experiment and uncertainties of the theoretical approaches used) between measured and calculated Stark FWHM values. We recommend the Stark FWHMs of the intense $254.182 \mathrm{~nm}, 308.624 \mathrm{~nm}$, and $309.342 \mathrm{~nm} \mathrm{Si} \mathrm{III,} \mathrm{and} 314.956 \mathrm{~nm}$ and also $316.571 \mathrm{~nm}$ Si IV lines for the plasma diagnostic purposes.
\end{abstract}

Key words. plasmas - line: profiles - atomic data - atomic processes

\section{Introduction}

The silicon ( $\mathrm{Si}$ ) atoms and ions are present in many kinds of cosmic plasmas. The neutral (Si I), singly (Si II), doubly (Si III), and triply (Si IV) ionized silicon spectral lines are discovered in the emission and absorption spectra emitted by various cosmic light sources. We mention a few recent articles emphasizing the role of the prominent Si I-IV spectral lines in various fields of the astrophysical plasma diagnostics.

Statistical equilibrium of neutral and ionized silicon in the solar photosphere has been investigated by Shi et al. (2008). The Si I line was discovered in the emission spectra of a $\mathrm{K}$ giants (Sundqvist et al. 2008). The equivalent widths of Si II $400 \mathrm{~nm}$ lines have been used for the determining diversity of supernovae Ia (Arsenijević et al. 2008). In the 500-700 nm wavelength interval, the SN Ia - defining Si II feature occurs (Bongard et al. 2008). Prominent Si II emission lines have been observed in the first helium nova V445 Puppis (Iijima \& Nakanishi 2008). Strong Si IV ion absorption has been identified in seven GRB host galaxies $(z=2-4)$ (Fox et al. 2008). The presence of Si III and Si IV is identified in the interstellar medium seen towards the nearby (170 pc) star HD 102065 (Nehme et al. 2008). Kawka et al. (2008) refer to the detection of Si IV in the spectra of the hot white dwarfs in the post-common-envelope binaries, Feige 24, EUVE J00720-317, and EUVE J2013+400, and to detection of Si III and Si IV in the spectra of BPM 6502. Some Si III lines have been observed in the spectrum of the rapidly rotating classical $\mathrm{T}$ Tauri star RY Tau (Gomez de Castro \& Verdugo 2007) and the wind kinematics were derived from the Si III line profiles. The Si III and $\mathrm{Si}$ IV ions, as absorbers, have been discovered in the low- $z$ $(z<0.4)$ intergalactic medium (Danforth \& Shull 2008). The emergence of a high-velocity broad-line outflow in a luminous quasar J105400.40+034801.2 is evident in ultraviolet Si IV absorption lines (Hamann et al. 2008). The Si III triplet at $455 \mathrm{~nm}$ are used by Catanzaro \& Leone (2008) to determine the spectroscopic variability of the effective temperature $\left(T_{\text {eff }}\right)$ in the hot pulsating star Beta Cephei. These lines, relatively insensitive to the variation in $T_{\text {eff }}$ through a pulsation cycle, show small equivalent width variations (Catanzaro et al. 2008).

Thesee references clearly confirm the recent astrophysical interest for the neutral and ionized silicon spectral line characteristics. In many models of astrophysical plasmas (Lesage 1994; Dimitrijević et al. 2007) the Stark width, beside the Doppler caused broadening, is essential. A summary of the papers dedicated to the Si I-IV Stark width calculations is available in NIST (2009), while the critical reviews of the measured values are given in Lesage (2009) and Konjević et al. (2002), and references therein.

The aim of this work is to present experimental Stark widths for a number of spectral lines from neutral, singly, doubly, and triply ionized silicon spectra emitted by the pulsed helium 
discharge, which is optically thin at the investigated ionic silicon wavelengths. After applying procedure proposed in the Appendix, we have found a weak self-absorption in Si I spectra with optical depth $<0.38$. Results for the Si I lines are corrected in the amount of $2-22 \%$. The specific design of the discharge tube provides the Si I, Si II, Si III, and Si IV spectra within the single pulse at the different stages of the discharge. Therefore, the Stark widths of different ionic species presented in this paper are for the first time measured in the essentially same laboratory plasma. The Stark FWHM (full-width at half of the maximum line intensity, $W$ ) values are obtained in the wavelength interval (206-640) $\mathrm{nm}$ at the electron densities between $3.7 \times 10^{22} \mathrm{~m}^{-3}$ and $1.1 \times 10^{23} \mathrm{~m}^{-3}$ and at the electron temperatures $(T)$ ranging from $12500 \mathrm{~K}$ to $19000 \mathrm{~K}$, both of which are interesting in astrophysics.

\section{Experiment}

A modified version of the linear low-pressure pulsed arc (Djeniže et al. 1992, 2002) has already been used as a plasma source (see Fig. 1 in Djeniže 2009). A pulsed discharge was produced in a pyrex discharge tube of $5 \mathrm{~mm}$ inner diameter with a plasma length of $12 \mathrm{~cm}$. The silicon atoms $(\mathrm{O}, \mathrm{B}$ and $\mathrm{Na}$ atoms, also) are evaporated from the axial part of the discharge tube. As a working gas helium $(90 \% \mathrm{He}+7 \% \mathrm{~N}+3 \% \mathrm{O})$ was used at $665 \mathrm{~Pa}$ flowing pressure. The helium metastables favor the ionization of the silicon atoms due to the Penning effect (Kruithof \& Penning 1937) ensuring a high concentration of the Si II ions.

The discharge is created using the capacitor of $14 \mu \mathrm{F}$ charged up to $44 \mathrm{~J}$ bank energy. Plasma reproducibility was monitored through the radiation originating from the chosen Si I, Si II, and Si III lines and discharge current using a Rogowski coil signal (Rogowski \& Steinhaus 1912). It was found that scatter of the peak value of the discharge current is within $\pm 4 \%$.

For the first time, we applied the McPherson model 209 spectrograph (1.33 m focal length) equipped with 2400 grooves/mm holographic grating, resulting in reciprocal linear dispersion of $0.28 \mathrm{~nm} / \mathrm{mm}$ in the first order. As a detection system the Andor DH740-18F-03 iStar intensified CCD camera is employed. The system was carefully calibrated using a set of pen-light ( $\mathrm{Ne}, \mathrm{Ar}$ and $\mathrm{Hg}$ ) sources produced by the LOT-Oriel. We found that instrumental profile of the spectrograph itself in the first order, conventionally measured (with 9789 QB EMI photomultiplier), corresponds to the Gaussian function of $F W H M=3.0 \mathrm{pm}$, while the overall profile (spectrograph + ICCD camera) can be approximated by the Voigt function with $F W H M$ of $8.5 \mathrm{pm}$ at $614.3 \mathrm{~nm}$. Standard light guide and focusing optics, employed at some stages of measurement, have no influence on the shape and width of the instrumental profile.

The spectroscopic observations were made end-on along the axis of the discharge tube. The camera was triggered at the specified moment with exposure time adapted to our discharge conditions $(0.5 \mu \mathrm{s})$. To reduce thermal noise the ICCD detector is always kept on $-25{ }^{\circ} \mathrm{C}$. Some of the recorded Si I, Si II, Si III, and Si IV spectral line profiles are presented in Figs. 1-4. It should be mentioned that, at our experimental conditions, the Si II 505.598/504.103 lines intensity ratio is more than twice higher than the value which follows from NIST (see Fig. 2c), but agrees with one predicted by transition probabilities and statistical weights related to the mentioned transitions (see Eq. (2) in Djeniže \& Bukvić 2001, and data in NIST).

The helium plasma parameters were determined using standard diagnostic methods; the electron density $(N)$ was estimated

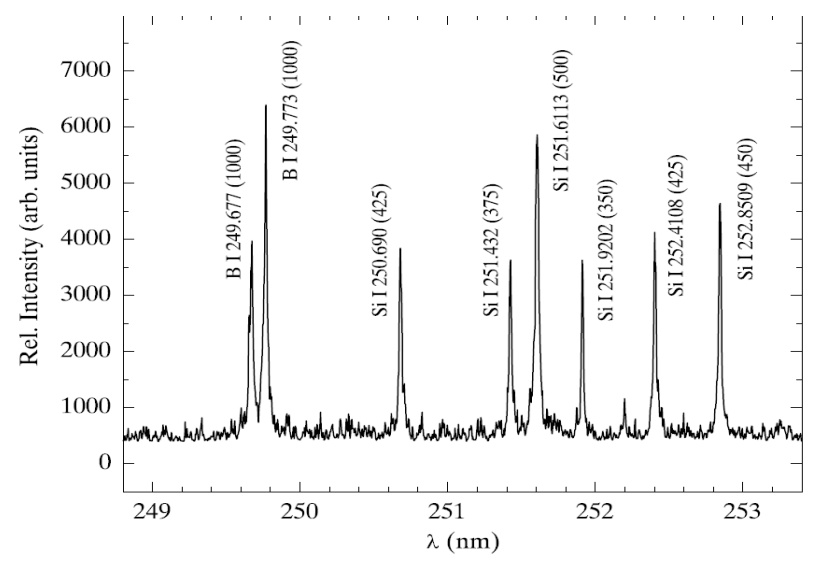

Fig. 1. The recorded resonance Si I line profiles (see Table 2). The numbers in brackets denote relative line intensities tabulated by NIST (2009). The resonance B I line profiles are also presented.

relying on the known Stark $F W H M$ of the He II $\mathrm{P}_{\alpha}(468.6 \mathrm{~nm})$ spectral line. It should be mentioned that the intensity of the He II $\mathrm{P}_{\alpha}$ drops down quickly, and after the 35 th $\mu$ s signal to noise ratio is not sufficient for accurate processing.

The electron temperature was obtained using the relative line intensity ratio method (Saha equation) (Griem 1964) between Si III $(308.62,309.34$, and $309.68 \mathrm{~nm})$ and Si IV (314.96 nm and $316.57 \mathrm{~nm})$, and also between O II (397.33 nm) and O III (396.16 nm) spectral lines with an estimated error of $\pm 14 \%$ assuming the local thermodynamic equilibrium (LTE) (Rompe \& Stenbeeck 1967), and taking the lowering of the ionization energy into account (Drawin \& Felenbok 1965). The necessary atomic data are taken from NIST (2009).

Temporal evolutions of the $N$ and $T$ are presented in Fig. 5. Recovery of the electron temperature and electron density at the 30th $\mu \mathrm{s}$ is due to second half period of the discharge current, caused by subcritically damped electrical circuit (see Fig. 5). Unexpectedly high value of $T$ at the 30th $\mu$ s could be attributed, to the some extent, to hot Penning electrons produced in collisions of two helium $2 \mathrm{~s}^{3} \mathrm{~S}_{1}$ metastables with $18.92 \mathrm{eV}$ excitation energy (Silfvast 1971).

The Si I and Si II line profiles are investigated at lower, while the Si III and Si IV line profiles at higher electron temperatures. The Stark FWHMs are obtained at various electron densities ranging between $3.7 \times 10^{22} \mathrm{~m}^{-3}$ and $1.1 \times 10^{23} \mathrm{~m}^{-3}$. Their values, normalized at an $N=1.0 \times 10^{23} \mathrm{~m}^{-3}$, are presented in Tables 2-5.

The spectral purity of the investigated silicon lines are checked using a list of the identified wavelengths given by NIST (2009).

\section{Line profile deconvolution procedure}

The measured Si II, Si III, and Si IV line profiles were of the Voigt type due to a convolution of the Lorentzian Stark, Gaussian profile caused by Doppler, and Voigt instrumental (spectrograph + ICCD) broadening. For the electron density and temperature in the presented experiment, the Lorentzian fraction was dominant. Van der Waals and resonance broadenings (Griem 1974) were estimated to be smaller by more than one order of magnitude than Stark, Doppler, and instrumental broadenings.

For estimation of spectral line parameters a deconvolution procedure (Davies \& Vaughan 1963) is applied based on the least-squares algorithm. Estimation of the spectrum baseline 

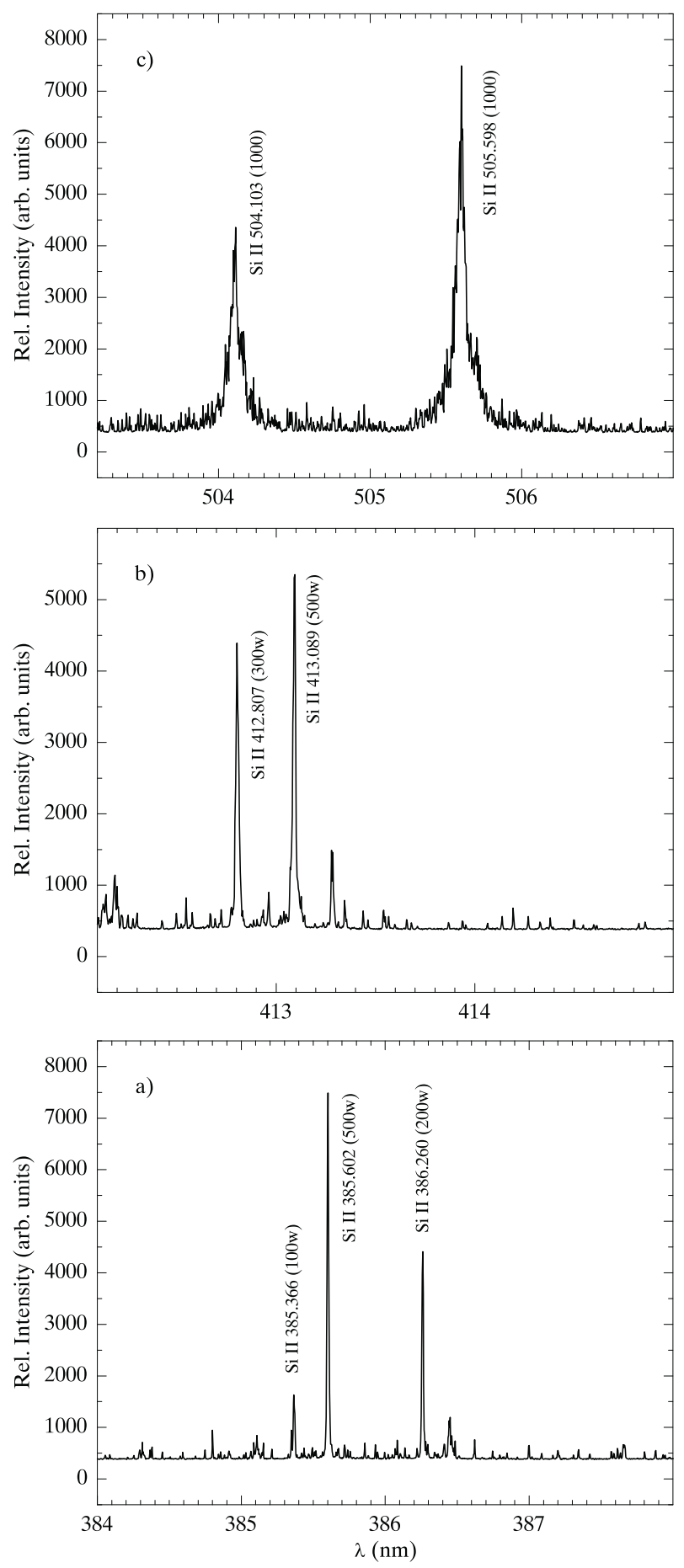

Fig. 2. Panels a-c) The recorded profiles of prominent Si II spectral lines present in many astrophysical spectra. The numbers in brackets denote relative line intensities tabulated by NIST.

is based on the procedure presented by Bukvić \& Spasojević (2005), and Bukvić et al. (2008). As an example a Voigt fit of $254.182 \mathrm{~nm} \mathrm{Si}$ III spectral line is presented in Fig. 6. For this, a very-well defined line profile, error of the Lorentz component is $\sim 10 \%$. In general, for ionic lines the error of the Lorentz component is between $10 \%$ and $20 \%$, while it is slightly higher for atomic lines, up to $30 \%$. Uncertainty of the Lorentz component for neutral silicon lines is additionally increased for a few percent due to corrections for self-absorption (see Tables 2-5).
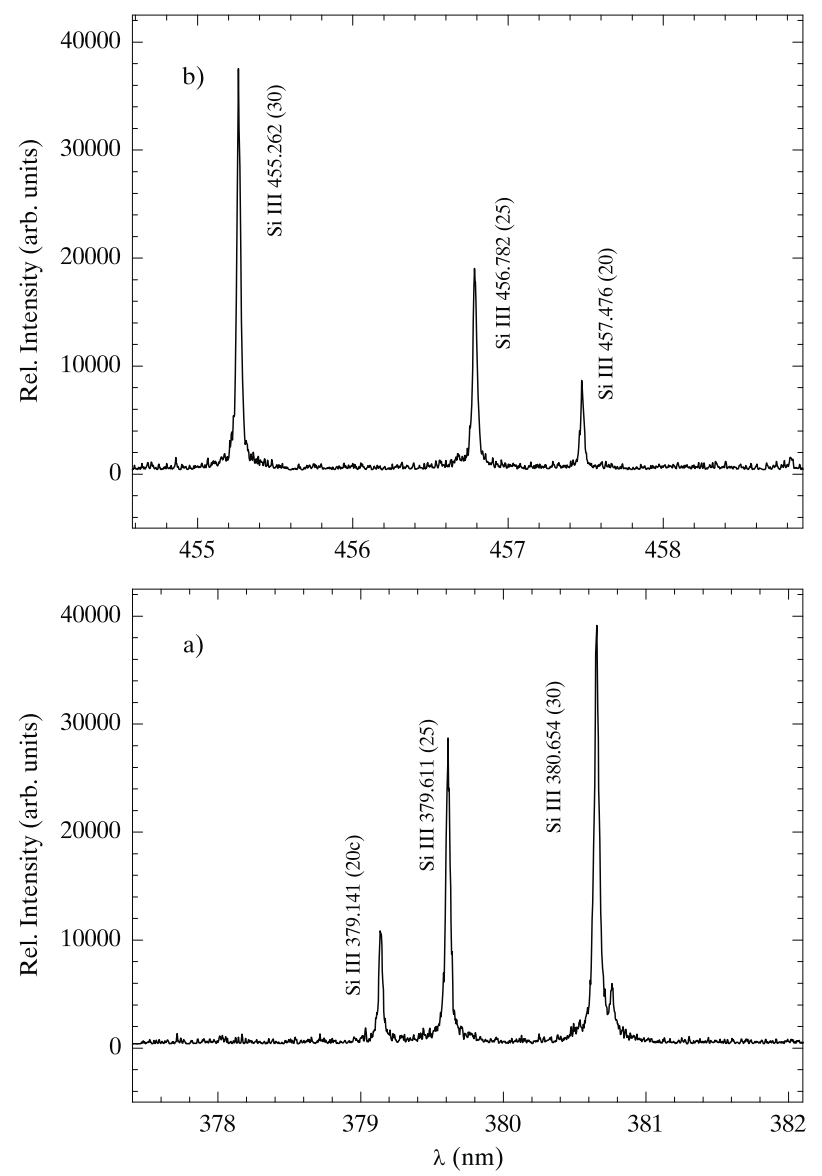

Fig. 3. Panels a) and b) The recorded profiles of prominent Si III spectral lines present in many astrophysical spectra. The numbers in brackets denote the relative line intensities tabulated by NIST.

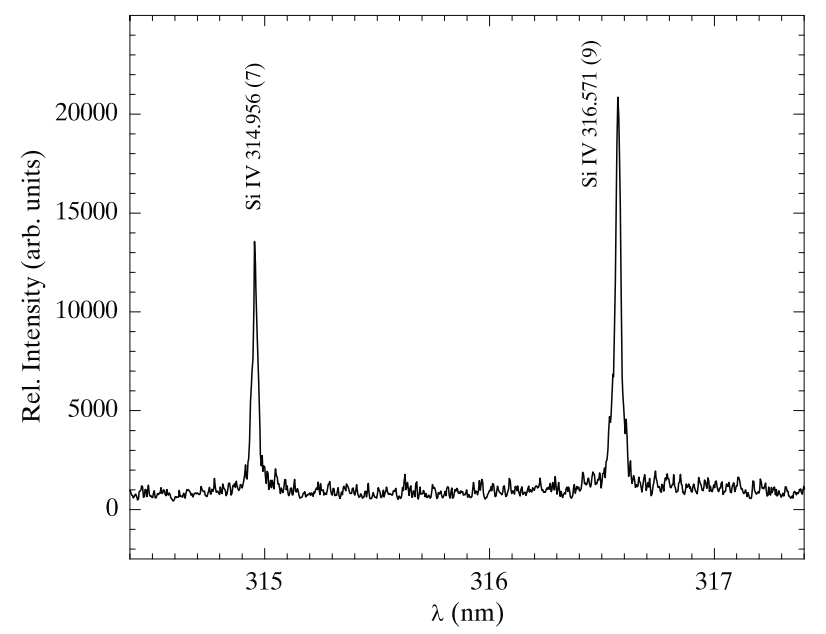

Fig. 4. The recorded profiles of the $314.956 \mathrm{~nm}$ and $316.571 \mathrm{~nm}$ $\mathrm{Si}$ IV spectral lines. The numbers in brackets denote relative line intensities tabulated by NIST.

\section{Results and discussion}

\subsection{Electron density measurement}

We mentioned above that the electron density $(N)$ was estimated by relying on the known Stark FWHM of the He II $\mathrm{P}_{\alpha}(468.6 \mathrm{~nm})$ spectral line. The inherent angle of view of the lens used to collect light was sufficient to cover whole cross section of the narrow part of the discharge tube. It means that the electron 


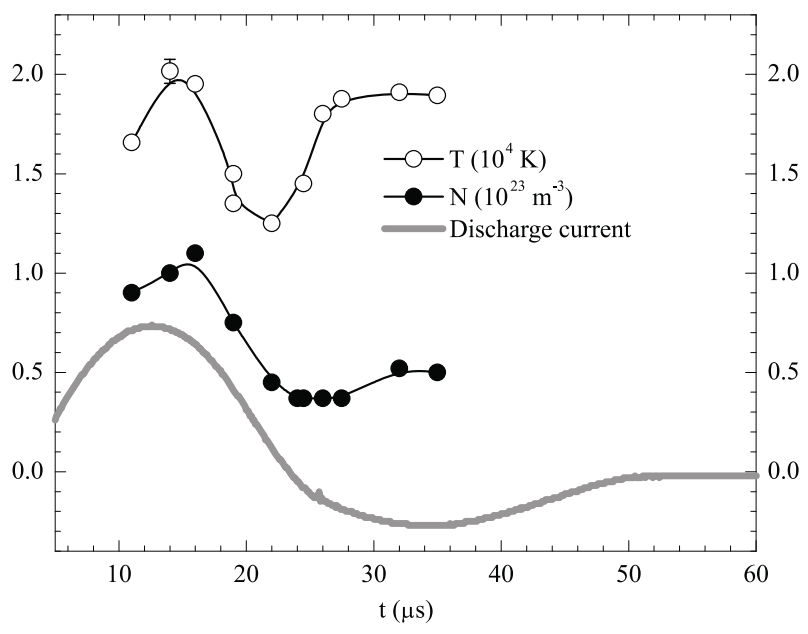

Fig. 5. Temporal evolutions of the electron density $\left(N\right.$ in $\left.10^{23} \mathrm{~m}^{-3}\right)$ and electron temperature ( $T$ in $10^{4} \mathrm{~K}$ ). The error bar represents $\pm 14 \%$ estimated uncertainties. The gray line is the discharge current (in arbitrary units) captured by the Rogowski coil.

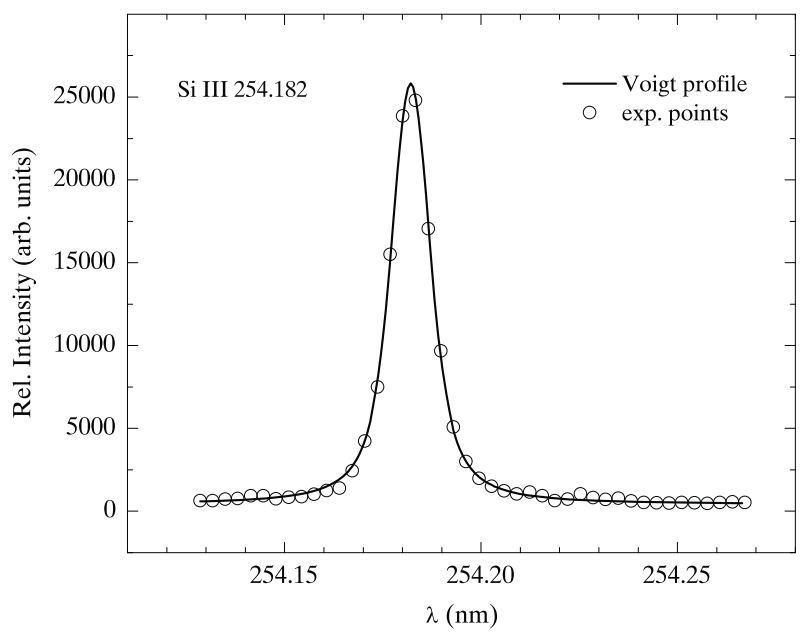

Fig. 6. The $254.182 \mathrm{~nm} \mathrm{Si} \mathrm{III} \mathrm{line} \mathrm{profile} \mathrm{recorded} \mathrm{at} N=5.2 \times 10^{22} \mathrm{~m}^{-3}$ and $T=19000 \mathrm{~K}$. Circles represent measured values. The solid line represents the corresponding Voigt profile using $4.4 \mathrm{pm}$ for the Gaussian parameter (Doppler + instrumental convolution) in the fitting procedure. The total FWHM $\left(W_{\mathrm{t}}\right)$ (Lorentzian + Gaussian convolution) is $12.02 \mathrm{pm}$ with the Stark component of $W_{\mathrm{m}}=3.4 \mathrm{pm}$ (see Table 4). The baseline estimation is made using the procedure described by Bukvić \& Spasojević (2005) and Bukvić et al. (2008).

density evaluated in this way is an average value with respect to the actual density distribution along the tube radius. To estimate electron density on the axis of the tube, we reduced the angle of view to capture light only from the central part of the discharge. In this case the monitored area is no more than $1 \mathrm{~mm}$ in radius, along the whole discharge tube. A drawback of such a tiny angle of view is a significant decrease of signal/noise ratio. We chose 10 th $\mu$ s to record $\mathrm{P}_{\alpha}$, the moment when the intensity of the line is still sufficient for reliable processing. According to our analysis, electron density measured on the axis of the tube is approximately $5 \%$ higher than the average value - the Lorentz FWHM of He II $\mathrm{P}_{\alpha}$ at the axis is $W_{a x}=0.402 \mathrm{~nm}$, while for the profile recorded integrally, the corresponding value is $W_{a v}=0.384 \mathrm{~nm}$.

In the second stage of our analysis we discuss one possible issue of the self-absorption. To accomplish a check for selfabsorption, we doubled the optical path length by placing a
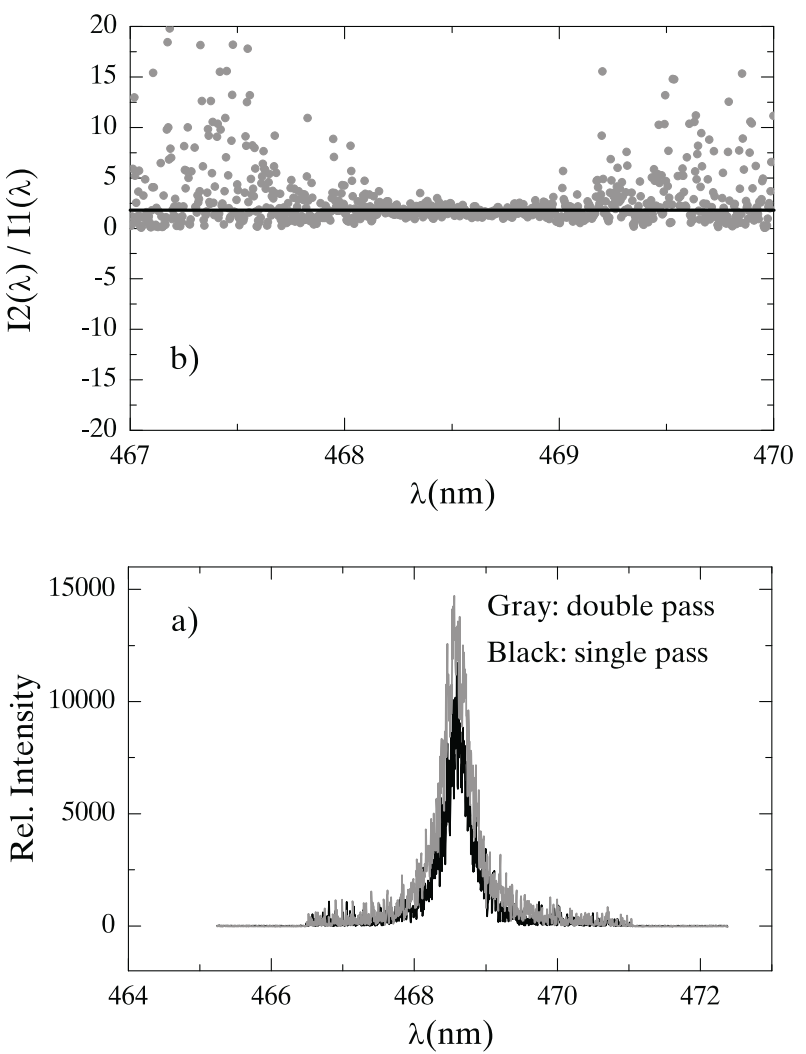

Fig. 7. Panel a): the He II $\mathrm{P}_{\alpha}$ line recorded in single pass (black) and in double pass (gray). Both profiles are taken at the 10th $\mu$ s and same detection conditions. The light is captured only from the central part of the discharge, $1 \mathrm{~mm}$ in radius. Intensity fluctuations are caused by a huge number of very low-intensity spectral lines superimposed on the ICCD noise. Panel b): the ratio of the two $\mathrm{P}_{\alpha}$ line profiles recorded in double (I2) and in single (I1) pass. In spite of noticeable scatter, specially on the line wings, it is easy to see that the main trend of the data is straight horizontal line. It means that the ratio of the two profiles is constant, i.e. self-absorption is not present.

concave aluminum mirror behind the plasma (Hutchinson 2002). Two He II $\mathrm{P}_{\alpha}$ profiles are subsequently recorded, with and without the auxiliary mirror. In Fig. 7 the corresponding $\mathrm{P}_{\alpha}$ profiles are presented together with their ratio after subtraction of the dark spectrum introduced by the ICCD. One can notice that there is no evidence of the self absorption and therefore optical depth for the $\lambda=468.6 \mathrm{~nm}$ can be neglected at the 10 th $\mu$ s when $\mathrm{P}_{\alpha}$ attains its maximum intensity ${ }^{1}$.

Finally, we consider an influence of the contiguous layers of plasma, on both sides of the central narrow tube, on the procedure for electron density estimation. In Fig. 8 two $\mathrm{P}_{\alpha}$ profiles are shown, the first one in gray is recorded along the discharge tube axis with angle of view set to collect light integrally, and it includes contributions of the both contiguous layers. The second profile originates from the light emitted only by one contiguous layer. It is recorded side-on, capturing emission of approximately the same volume that contributes in the axial view. By subtracting contribution of both contiguous layers from the

\footnotetext{
${ }^{1}$ Uncertainty of the optical depth estimated by this method is proportional to $\sigma / \sqrt{N}$ where $\sigma$ is the standard deviation of the data scatter and $N$ number of data points. Because of the nonsymmetric scatter we applied recently developed DLS method for data fitting (Bukvić et al. 2008) to estimate relevant quantities. It follows that the uncertainty of the optical depth is $\sim 0.009$. In other words, the maximum optical depth consistent with our data is $\leq 0.009$.
} 


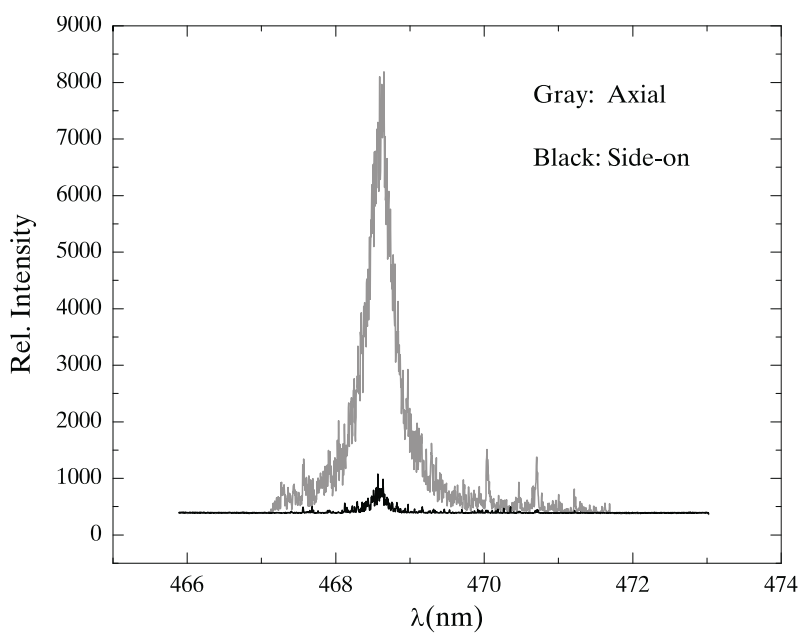

Fig. 8. The profile of the He II $\mathrm{P}_{\alpha}$ line recorded axially (gray) at the 10 th $\mu$ s from the beginning of the discharge. The black profile originates from the light emitted only by the one contiguous layer. It is recorded side-on, at the same moment capturing emission of approximately the same volume that contributes in the axial view.

profile recorded axially, one can get corrected $\mathrm{P}_{\alpha}$ profile, the basis for electron density estimation inside the narrow tube. The corrected profile is $\sim 6 \%$ wider, and the Lorentz FWHM is $W_{\text {corr }}=0.436 \mathrm{~nm}$, which results in $\sim 7 \%$ higher electron density. Within this paper we have adopted corrected electron density values estimated from integral axial observations with uncertainties less than $15 \%$ due to errors related to the averaging over the tube radius and contribution of the contiguous plasma layers. Error of the best fit Lorentz FWHM of the $\mathrm{P}_{\alpha}$ is low, about $1 \%$, owing to a large number of data points in the line profile.

\subsection{The Si I spectrum}

Radial distribution of the Si I emitters should be close to the uniform, meaning the line profiles of Si I spectra are based on integral observations along the axis of the discharge tube. A certain amount of asymmetry in the Si I spectral line profiles is to be expected due to interaction of neutral silicon atoms with Si II, He II, and O II ions. In our experimental conditions, this effect is masked by overall noise present in the Si I spectra. Consequently, the Si I spectral lines are processed as symmetric lines. In Fig. 9 we present the best fit of the Si I $251.920 \mathrm{~nm}$ line to the Voigt profile, together with residuals. One can notice that data scatter completely mask the expected asymmetry of the line profile. Therefore, we were not able to isolate the ionic contribution to the line broadening. Because of that, values quoted in Table 2 represent the overall Stark FWHM $\left(W_{\mathrm{m}}\right)$.

Since the silicon is introduced in the working gas by sputtering of the glass one can expect generally low density of the silicon atoms. On the other hand, the investigated spectral lines of neutral silicon belong to the resonance transitions, so the issue of self-absorption has to be considered. Standard procedure for self-absorption check (Hutchinson 2002), applied in analysis of the He II $\mathrm{P}_{\alpha}$, is inefficient here because the $\mathrm{Si} \mathrm{I}$ lines are narrow, and corresponding profiles are recorded in no more than 10 points. Also, scatter of the data points here is more pronounced than for He I-II lines. Because of that it is hard to conclude whether two profiles, recorded with and without the auxiliary mirror, are proportional to the same factor in each point.

An independent procedure is employed to check for selfabsorption. The essence is to calculate ratio of the measured

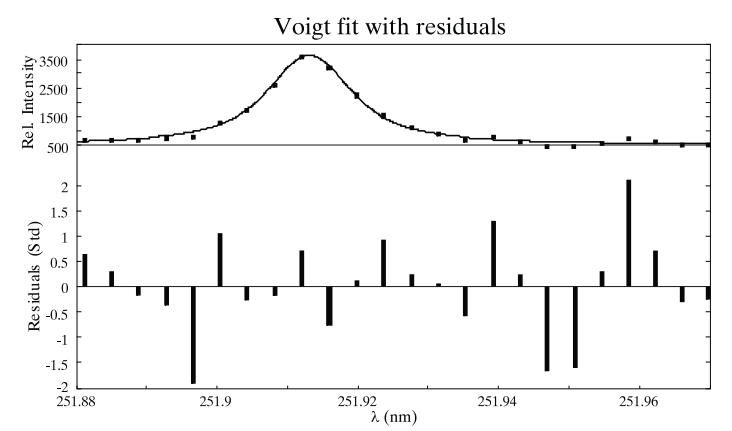

Fig. 9. The neutral silicon $\lambda=251.920 \mathrm{~nm}$ spectral line, together with the best fit Voigt function. Expected asymmetry of the line is masked by the data scatter, so that the residuals are evenly distributed around the model function.

Table 1. Quotients $Q$ for set of the six Si I spectral lines.

\begin{tabular}{|c|c|c|c|c|c|c|}
\hline$p$ & $\overline{250.690}$ & $\overline{251.432}$ & $\overline{251.611}$ & $\overline{251.920}$ & $\overline{252.411}$ & 252.851 \\
\hline$q$ & & & & & & \\
\hline 250.690 & 1 & & & & & \\
\hline 251.432 & 0.87 & 1 & & & & \\
\hline 251.611 & 1.18 & 1.33 & 1 & & & \\
\hline 251.920 & - & - & - & 1 & & \\
\hline 252.411 & - & - & - & 0.95 & 1 & \\
\hline 252.851 & - & - & - & 0.95 & 1.18 & 1 \\
\hline
\end{tabular}

Note 1 . The quotients $Q_{p, q}=R_{p, q}^{\mathrm{m}} / R_{p, q}^{\mathrm{th}}$ of the measured intensity ratios $R_{p, q}^{\mathrm{m}}=I_{p} / I_{q}$ and theoretical $R_{p, q}^{\mathrm{th}}=A_{p} g_{p} / A_{q} g_{q}$ for the six Si I spectral lines from the resonance $3 \mathrm{~s}^{2} 3 \mathrm{p}^{2}-3 \mathrm{~s}^{2} 3 \mathrm{p} 4 \mathrm{~s}$ transition. The transition probabilities $(A)$ and statistical weights $(g)$ are taken from NIST (2009). Every cell contains quotient $Q_{p, q}$ calculated for particular pair of spectral lines $p$ and $q$, measured at the same temperature. The first group of three lines $(250.690 \mathrm{~nm}, 251.432 \mathrm{~nm}, 251.611 \mathrm{~nm})$ is recorded at $T=15000 \mathrm{~K}$, the second group $(251.920 \mathrm{~nm}, 252.411 \mathrm{~nm}$, $252.851 \mathrm{~nm}$ ) is recorded at $T=12500 \mathrm{~K}$.

intensities for two spectral lines, $p$ and $q$, belonging to the same transition $\left(R_{p, q}^{\mathrm{m}}=I_{p} / I_{q}\right)$ and to compare it to the ratio $R_{p, q}^{\text {th }}=A_{p} g_{p} / A_{q} g_{q}$ where $A$ denotes the transition probability while $g$ is the statistical weight. Ideally the both ratios should be the same, if the plasma is free of self-absorption. Because of the experimental errors associated to the line intensities (typically $\sim 6 \%$ ) and uncertainties of the transition probabilities (mark C, up to the $\sim 25 \%$ in NIST 2009) the ratios will differ. Table 1 summarizes quotients of both ratios $\left(Q_{p, q}=R_{p, q}^{\mathrm{m}} / R_{p, q}^{\mathrm{th}}\right)$ for six Si I lines given in Fig. 1. One can notice that the worst disagreement is $33 \%$, still within the margin imposed by experimental errors and uncertainties of the tabulated $A$ values. Applying additional analysis presented in Appendix we have found that optical depth $k_{\lambda} l$ for considered spectral lines is in the range $0.07<k_{\lambda} l<0.38$. Corresponding Stark widths are subsequently corrected to the limit of an optically thin layer (see Table 2).

To check the contribution of the plasma layers beyond the narrow part of the discharge tube, we made the side-on recordings of the spectral regions with the most intense Si I lines. According to our findings, the intensity of the Si I lines originating from contiguous plasma layers is under the detectible limit, therefore their contributions to the profiles recorded axially can be neglected.

Our experimental results are presented in Table 2, together with existing results published by various authors. Three experiments are dedicated to investigating the resonance Si I lines 
Table 2. The Si I experimental Stark widths.

\begin{tabular}{|c|c|c|c|c|c|c|c|c|c|}
\hline Transition & $\lambda(\mathrm{nm})$ & $T$ & $\overline{W_{\mathrm{m}}}$ & $W_{\mathrm{m}}^{\text {corr }}$ & $W_{\mathrm{m}} / W_{\mathrm{th}}$ & $W_{\mathrm{m}}^{\mathrm{corr}} / W_{\mathrm{th}}$ & $\mathrm{a}$ & $\mathrm{b}$ & $\overline{\mathrm{c}}$ \\
\hline $3 \mathrm{~s}^{2} 3 \mathrm{p}^{2}{ }^{3} \mathrm{P}_{0}-3 \mathrm{~s} 3 \mathrm{p}^{3}{ }^{3} \mathrm{D}_{1}^{\circ}$ & 220.798 & 12.5 & $7.2 \pm 2.2$ & $6.5 \pm 2.2$ & & & & & \\
\hline $3 s^{2} 3 p^{2}{ }^{3} P_{1}-3 s 3 p^{3}{ }^{3} D_{2}^{0}$ & 221.089 & 12.5 & $7.8 \pm 2.3$ & $6.1 \pm 2.3$ & & & & & \\
\hline $3 \mathrm{~s}^{2} 3 \mathrm{p}^{2}{ }^{3} \mathrm{P}_{1}-3 \mathrm{~s}^{2} 3 \mathrm{p} 4 \mathrm{~s}^{3} \mathrm{P}_{2}^{\circ}$ & 250.690 & 15.0 & $14.5 \pm 4.1$ & $14.1 \pm 4.1$ & 1.43 & 1.39 & 70 & 28 & \\
\hline $3 \mathrm{~s}^{2} 3 \mathrm{p}^{2}{ }^{3} \mathrm{P}_{0}-3 \mathrm{~s}^{2} 3 \mathrm{p} 4 \mathrm{~s}^{3} \mathrm{P}_{1}^{\circ}$ & 251.432 & 15.0 & $11.5 \pm 3.2$ & $11.2 \pm 3.2$ & 1.13 & 1.10 & & 30 & \\
\hline $3 s^{2} 3 p^{2}{ }^{3} P_{2}-3 s^{2} 3 p 4 s^{3} P_{2}^{\circ}$ & 251.611 & 15.0 & $12.7 \pm 3.6$ & $11.7 \pm 3.6$ & 1.25 & 1.15 & & 30 & \\
\hline $3 \mathrm{~s}^{2} 3 \mathrm{p}^{2}{ }^{3} \mathrm{P}_{1}-3 \mathrm{~s}^{2} 3 \mathrm{p} 4 \mathrm{~s}^{3} \mathrm{P}_{1}^{\circ}$ & 251.920 & 12.5 & $12.4 \pm 3.5$ & $11.2 \pm 3.5$ & 1.26 & 1.13 & & 28 & \\
\hline $3 s^{2} 3 p^{2}{ }^{3} \mathrm{P}_{1}-3 s^{2} 3 p 4 s^{3} \mathrm{P}_{0}^{\circ}$ & 252.411 & 12.5 & $11.9 \pm 3.3$ & $10.4 \pm 3.4$ & 1.20 & 1.05 & 72 & 28 & \\
\hline $3 s^{2} 3 p^{2}{ }^{3} \mathrm{P}_{2}-3 s^{2} 3 p 4 s^{3} \mathrm{P}_{1}^{\circ}$ & 252.851 & 12.5 & $13.0 \pm 3.6$ & $10.7 \pm 3.7$ & 1.31 & 1.07 & 68 & 30 & \\
\hline $3 \mathrm{~s}^{2} 3 \mathrm{p}^{2}{ }^{1} \mathrm{D}_{2}-3 \mathrm{~s}^{2} 3 \mathrm{p} 4 \mathrm{~s}^{1} \mathrm{P}_{1}^{\mathrm{o}}$ & 288.158 & 15.0 & $14.5 \pm 4.1$ & & 1.04 & & 54 & 34 & 62 \\
\hline $3 \mathrm{~s}^{2} 3 \mathrm{p}^{2}{ }^{1} \mathrm{D}_{2}-3 \mathrm{~s}^{2} 3 \mathrm{p} 4 \mathrm{~s}^{3} \mathrm{P}_{1}^{\mathrm{o}}$ & 298.764 & 15.0 & $14.7 \pm 4.1$ & & & & & & \\
\hline $3 s^{2} 3 p^{2} 7{ }^{1} \mathrm{~S}_{0}-3 s^{2} 3 p 4 s s^{1} \mathrm{P}_{1}^{\circ}$ & 390.552 & 12.5 & $20.8 \pm 5.8$ & & 0.85 & & & 32 & 18 \\
\hline The transition is not given & 206.119 & 15.0 & $5.0 \pm 1.5$ & & & & & & \\
\hline The transition is not given & 243.515 & 12.5 & $12.5 \pm 3.5$ & & & & & & \\
\hline
\end{tabular}

Note 2. Our measured Si I Stark $F W H M\left(W_{\mathrm{m}}\right.$ in pm) at given $T$ (in $\left.10^{3} \mathrm{~K} \pm 14 \%\right)$ and $N=1 \times 10^{23} \mathrm{~m}^{-3} \pm 13 \%$, with estimated accuracies. $W_{\mathrm{m}}^{\text {corr }}$ denotes Stark $F W H M$ values after corrections for self-absorption. The $W$ (in pm) values from other experiments are also given: a (Srećković et al. 1998); b (Purić et al. 1974) and c (Meyer \& Beck 1970) obtained at following $T$ and $N$ : a $\left(T=28500 \mathrm{~K}, N=1.9 \times 10^{23} \mathrm{~m}^{-3}\right)$; b $(T=16400 \mathrm{~K}$, $\left.N=1.0 \times 10^{23} \mathrm{~m}^{-3}\right)$, and $\mathrm{c}\left(T=12200 \mathrm{~K}, N=1.0 \times 10^{23} \mathrm{~m}^{-3}\right)$. $W_{\text {th }}$ represents values presented by Griem (1974), calculated for electrons as perturbers only. Expected corrections for quasi-static broadening by ions, extrapolated from Griem (1974, p. 343), is roughly about $10 \%$ at the high densities in the experiment. Atomic data are taken from NIST.

$W$ values (see Table 2), no one is made in helium plasma. Eight Si I $W$ values for high lying transitions (with $\lambda>500 \mathrm{~nm}$ ), not researched in this work, are measured by Miller \& Bengtson (1970). A review of calculated Si I $W$ values is given in Griem (1974).

It is to be pointed out that the Si I spectrum has low intensity and is available only in a narrow window, between 22 th $\mu$ s and 24 th $\mu$ s after the beginning of the discharge, which corresponds to the noticeable minima in the electron temperature and electron density flow. We already mentioned that the minima of $T$ and $N$ appear at the moment when the discharge current changes its sign and has a low magnitude, see Fig. 5. Outside of this tiny window, the Si I line intensities are very low and the Si I spectrum is overpowered by Si III and Si IV spectra. Our new acquisition set-up provides accurate measurement of low-intensity spectral lines, and therefore Si I $W$ values are more accurate than results by previous experimental technique.

\subsection{The Si Il spectrum}

It should be mentioned that in the last four decades Si II was the fourth most experimentally investigated emitter, after hydrogen, helium, and argon. Therefore, the Si II Stark widths have been measured by many authors using different plasma sources (Konjević et al. 1970; Purić et al. 1974; Lesage et al. 1977; Chiang \& Griem 1978; Lesage et al. 1983; Kusch \& Schröder 1982; Peréz et al. 1993; Wollschläger et al. 1997; Gonzalez et al. 2002; Lesage \& Redon 2004). The critical reviews of the published experimental Stark widths are presented by Lesage (2009) and Konjević et al. (2002, and references therein).

The calculated Si II $W$ values (based on various theoretical approximations) are presented by Sahal-Bréchot (1968), Griem (1974) and Lanz et al. (1988). Our experimental Stark FWHMs $\left(W_{\mathrm{m}}\right)$ are presented in Table 3 , side by side with nine results published in the last forty years. The Stark FWHMs dependencies on the electron temperature for the most investigated Si II line profiles are presented in Fig. 10.

Here presented Si II $W$ values agree with ones calculated by Lanz et al. (1988) for the most investigated transitions (see Fig. 10). It should be mentioned that at electron temperatures below $15000 \mathrm{~K}$ values calculated by Lanz et al. (1988) and values presented by Griem (1974) differ for $25 \%$ for the higher Si II multiplets. In the multiplet No. 1, the LDA $W$ values are significantly below $G$ values (see Fig. 10a), while our $W_{\mathrm{m}}$ values agree with the calculated LDA $W$ values.

The Si II spectrum, as for Si I, is visible at the moment when $N$ and $T$ have minimal values during the discharge. However, the time window for observation is almost twice wider, from 21th $\mu \mathrm{s}$ up to 26 th $\mu$ s.

\subsection{The Si III spectrum}

The Si III lines are the most intense spectral lines observed in our helium plasma source at this discharge condition. In particular, the well isolated $254.182 \mathrm{~nm} \mathrm{Si}$ III line has been found to be the most intense during the whole plasma decay interval. It is also the most narrow among the investigated Si III lines (see Fig. 6 and Table 4). This line originates from the $3 \mathrm{p}^{2}{ }^{1} \mathrm{D}_{2}$ $\mathrm{Si}$ III level with a $15.153 \mathrm{eV}$ excitation energy, and represents a single allowed spontaneous de-excitation channel from this level (NIST 2009). Our measured Stark FWHMs $\left(W_{\mathrm{m}}\right)$ are presented in Table 4, together with other authors' results.

Some Si III $W$ values were calculated by Dimitrijević \& Konjević (1981) in the 3p-4s, 4s-4p, 3s-4p, 4p-4d, and 4p$5 \mathrm{~s}$ transitions. Our Si III $W_{\mathrm{m}}$ values for ten lines show the best agreement (within 20\%) with the calculated ones based on the modified semiempirical approximation.

\subsection{The Si IV spectrum}

The Si IV $W$ values were experimentally investigated in two works and calculated by Dimitrijević et al. (1991). Our measured Stark FWHMs $\left(W_{\mathrm{m}}\right)$ are presented in Table 5, together with other authors' results.

Direct comparison of our results with calculated values presented by Dimitrijević et al. (1991) is not possible due to the significant difference in electron temperatures. However, the values estimated by extrapolation indicate that, for the majority of lines, calculated Stark parameters are higher, about $27 \%$ in average, compared to our data. For two Si IV spectral lines $(212.018 \mathrm{~nm}$ 
Table 3. The measured Si II Stark widths.

\begin{tabular}{|c|c|c|c|c|c|c|c|c|c|c|c|c|}
\hline Transition & $\lambda(\mathrm{nm})$ & $\bar{T}$ & $\overline{W_{\mathrm{m}}}$ & $\overline{\mathrm{d}}$ & $\overline{\mathrm{e}}$ & $\bar{f}$ & $\overline{\mathrm{g}}$ & $\overline{\mathrm{h}}$ & $\overline{\mathrm{i}}$ & $\bar{j}$ & $\overline{\mathrm{k}}$ & $\mathrm{Zz}$ \\
\hline $3 \mathrm{~s} 3 \mathrm{p}^{2}{ }^{2} \mathrm{D}_{3 / 2}-3 \mathrm{~s}^{2} 4 \mathrm{f}^{2} \mathrm{~F}_{5 / 2}^{\circ}$ & 207.202 & 15.6 & $12.0 \pm 3.4$ & & & & & & & & & \\
\hline $3 \mathrm{~s} 3 \mathrm{p}^{2}{ }^{2} \mathrm{D}_{5 / 2}-3 \mathrm{~s}^{2} 4 \mathrm{f}^{2} \mathrm{~F}_{7 / 2}^{\mathrm{o}}$ & 207.270 & 15.6 & $10.0 \pm 3.0$ & & & & & & & & & \\
\hline $3 s^{2} 3 d^{2} D_{3 / 2}-3 s^{2} 5 f^{2} F_{5 / 2}^{o}$ & 290.428 & 15.6 & $311 \pm 62$ & & & & & & & & & \\
\hline $3 s^{2} 3 d^{2} D_{5 / 2}-3 s^{2} 5 f^{2} F_{7 / 2}^{o}$ & 290.569 & 15.6 & $349 \pm 70$ & & & & & & & & & \\
\hline $3 s 3 p^{2}{ }^{2} D_{3 / 2}-3 s^{2} 4 p^{2} P_{3 / 2}^{\circ}$ & 385.366 & 15.0 & $53.5 \pm 9.2$ & 52 & 57 & & & & & & & \\
\hline $3 s 3 p^{2}{ }^{2} D_{5 / 2}-3 s^{2} 4 p^{2} P_{3 / 2}^{\circ}$ & 385.602 & 15.0 & $49.0 \pm 6.8$ & 50 & 51 & & 68 & & 107 & 100 & 56 & 73 \\
\hline $3 \mathrm{~s} 3 \mathrm{p}^{2}{ }^{2} \mathrm{D}_{3 / 2}-3 \mathrm{~s}^{2} 4 \mathrm{p}^{2} \mathrm{P}_{1 / 2}^{\circ}$ & 386.260 & 15.0 & $43.0 \pm 6.8$ & 50 & 51 & & 68 & & 105 & 98 & 48 & 76 \\
\hline $3 s^{2} 3 d^{2} D_{3 / 2}-3 s^{2} 4 f^{2} F_{5 / 2}^{\circ}$ & 412.807 & 15.0 & $105 \pm 11$ & 97 & & 131 & 96 & & 158 & & & \\
\hline $3 s^{2} 3 d^{2} D_{5 / 2}-3 s^{2} 4 f^{2} F_{7 / 2}^{\circ}$ & 413.089 & 15.0 & $120 \pm 14$ & 101 & & 147 & 96 & & 160 & & & \\
\hline $3 s^{2} 4 p^{2} \mathrm{P}_{1 / 2}^{\circ}-3 s^{2} 4 d^{2} D_{3 / 2}$ & 504.103 & 15.0 & $229 \pm 45$ & 254 & & 164 & 242 & 390 & 253 & & & \\
\hline $3 s^{2} 4 p^{2} P_{3 / 2}^{\circ}-3 s^{2} 4 d^{2} D_{5 / 2}$ & 505.598 & 15.0 & $250 \pm 50$ & 258 & & 204 & 240 & 453 & 269 & & & \\
\hline $3 s^{2} 4 p^{2} \mathrm{P}_{1 / 2}^{\circ}-3 s^{2} 5 s^{2} S_{1 / 2}$ & 595.756 & 12.5 & $292 \pm 58$ & 272 & & & 304 & 369 & 278 & & & \\
\hline $3 s^{2} 4 p^{2} P_{3 / 2}^{\circ}-3 s^{2} 5 s^{2} S_{1 / 2}$ & 597.893 & 12.5 & $276 \pm 55$ & 278 & & & & 408 & 275 & & & \\
\hline $3 s^{2} 4 s^{2} S_{1 / 2}-3 s^{2} 4 p^{2} P_{3 / 2}^{\circ}$ & 634.710 & 12.5 & $185 \pm 37$ & 113 & & 125 & 124 & & 196 & 214 & & 244 \\
\hline $3 s^{2} 4 s^{2} S_{1 / 2}-3 s^{2} 4 p^{2} P_{1 / 2}^{o / 2}$ & 637.136 & 12.5 & $187 \pm 37$ & 99 & & 123 & 110 & & 193 & 222 & 82 & 234 \\
\hline
\end{tabular}

Note 3. Our measured Si II Stark FWHM ( $W_{\mathrm{m}}$ in pm) at given $T$ (in $10^{3} \mathrm{~K} \pm 14 \%$ ) and $N=1 \times 10^{23} \mathrm{~m}^{-3} \pm 13 \%$, with estimated accuracies. The $W$ (in pm) values from other experiments are also given: d (Gonzalez et al. 2002), e (Wollschläger et al. 1997), f (Peréz et al. 1993), g (Lesage et al. 1983), h (Kusch \& Schröder 1982), i (Lesage et al., 1977), j (Chiang \& Griem 1978), k (Purić et al. 1974), and z (Konjević et al. 1970) obtained at the following $T$ and $N: \mathrm{d}\left(T=18000 \mathrm{~K}, N=1.0 \times 10^{23} \mathrm{~m}^{-3}\right)$, e $\left(T=14300 \mathrm{~K}, N=1.0 \times 10^{23} \mathrm{~m}^{-3}\right), \mathrm{f}\left(T=16400 \mathrm{~K}, N=1.0 \times 10^{23} \mathrm{~m}^{-3}\right)$, $\mathrm{g}\left(T=16400 \mathrm{~K}, N=1.0 \times 10^{23} \mathrm{~m}^{-3}\right), \mathrm{h}\left(T=16400 \mathrm{~K}, N=1.0 \times 10^{23} \mathrm{~m}^{-3}\right), \mathrm{i}\left(T=16400 \mathrm{~K}, N=1.0 \times 10^{23} \mathrm{~m}^{-3}\right), \mathrm{j}(T=16400 \mathrm{~K}$, $\left.N=1.0 \times 10^{23} \mathrm{~m}^{-3}\right), \mathrm{k}\left(T=16400 \mathrm{~K}, N=1.0 \times 10^{23} \mathrm{~m}^{-3}\right)$, and $\mathrm{z}\left(T=8500 \mathrm{~K}, N=1.82 \times 10^{23} \mathrm{~m}^{-3}\right)$. Atomic data are taken from NIST.

Table 4. The measured Si III Stark widths.

\begin{tabular}{|c|c|c|c|c|c|c|c|c|}
\hline Transition & $\lambda(\mathrm{nm})$ & $T$ & $W_{\mathrm{m}}$ & $\mathrm{m}$ & $\mathrm{n}$ & $\mathrm{p}$ & $\mathrm{q}$ & $\mathrm{r}$ \\
\hline $3 \mathrm{~s} 3 \mathrm{p}^{1} \mathrm{P}_{1}^{\circ}-3 \mathrm{p}^{2}{ }^{1} \mathrm{D}_{2}$ & 254.182 & 19.0 & $6.5 \pm 0.6$ & 30 & & & & \\
\hline $3 \mathrm{~s} 3 \mathrm{~d}^{1} \mathrm{D}_{2}-3 \mathrm{p} 3 \mathrm{~d}{ }^{1} \mathrm{D}_{2}^{\circ}$ & 254.609 & 19.0 & $6.8 \pm 1.4$ & & & & & \\
\hline $3 \mathrm{~s} 3 \mathrm{~d}^{1} \mathrm{D}_{2}-3 \mathrm{~s} 4 \mathrm{f}^{1} \mathrm{~F}_{3}^{\circ}$ & 255.921 & 19.0 & $6.8 \pm 1.4$ & & & & & \\
\hline $3 p 3 d{ }^{1} D_{2}^{\circ}-3 p 4 p{ }^{1} P_{1}$ & 264.079 & 19.0 & $24.2 \pm 4.8$ & & & & & \\
\hline $3 s 4 f^{3} F_{2}^{\circ}-3 s 6 g{ }^{3} G_{3}$ & 303.473 & 18.8 & $17.5 \pm 3.5$ & & & & & \\
\hline $3 \mathrm{~s} 3 \mathrm{~d}^{3} \mathrm{D}_{3}-3 \mathrm{~s} 4 \mathrm{p}^{3} \mathrm{P}_{2}^{\circ}$ & 308.624 & 18.8 & $31.1 \pm 3.5$ & & & & & \\
\hline $3 \mathrm{~s} 3 \mathrm{~d}^{3} \mathrm{D}_{2}-3 \mathrm{~s} 4 \mathrm{p}^{3} \mathrm{P}_{1}^{\circ}$ & 309.342 & 18.8 & $28.5 \pm 3.0$ & & & & & \\
\hline $3 s 3 d^{3} D_{1}-3 s 4 p{ }^{3} P_{0}^{\circ}$ & 309.683 & 18.8 & $27.7 \pm 3.0$ & 29 & & & & \\
\hline $3 \mathrm{~s} 4 \mathrm{~d}^{1} \mathrm{D}_{2}-3 \mathrm{p} 3 \mathrm{~d}{ }^{1} \mathrm{P}_{1}^{\circ}$ & 318.513 & 18.8 & $27.7 \pm 5.5$ & & & & & \\
\hline $3 \mathrm{p} 3 \mathrm{~d}^{3} \mathrm{~F}_{3}^{\circ}-3 \mathrm{~s} 5 \mathrm{~g}^{3} \mathrm{G}_{4}$ & 319.650 & 18.8 & $32.3 \pm 6.5$ & & & & & \\
\hline $3 \mathrm{~s} 4 \mathrm{p}^{3} \mathrm{P}_{0}^{\circ}-3 \mathrm{~s} 5 \mathrm{~s}^{3} \mathrm{~S}_{1}$ & 323.050 & 19.0 & $43.3 \pm 8.6$ & & & & 18 & \\
\hline $3 s 4 p{ }^{3} P_{1}^{\circ}-3 s 5 s{ }^{3} S_{1}$ & 323.395 & 19.0 & $44.6 \pm 8.9$ & & & & 19 & \\
\hline $3 s 4 p{ }^{3} P_{2}^{\circ}-3 s 5 s{ }^{3} S_{1}$ & 324.162 & 19.0 & $48.1 \pm 9.6$ & 73 & & & 19 & \\
\hline $3 \mathrm{~s} 4 \mathrm{~d}^{3} \mathrm{D}_{2}-3 \mathrm{~s} 5 \mathrm{f}^{3} \mathrm{~F}_{3}^{\circ}$ & 348.691 & 19.0 & $50.2 \pm 10.0$ & & & & & \\
\hline $3 s 4 p{ }^{1} P_{1}^{\circ}-3 s 4 d{ }^{1} D_{2}$ & 359.047 & 18.8 & $86.6 \pm 17.3$ & & & & & \\
\hline $3 s 4 p^{3} P_{0}^{\circ}-3 s 4 d^{3} D_{1}$ & 379.141 & 18.8 & $74.3 \pm 13.8$ & & 68 & 54 & 20 & \\
\hline $3 s 4 p^{3} P_{1}^{\circ}-3 s 4 d^{3} D_{2}$ & 379.611 & 18.8 & $79.7 \pm 13.9$ & & 68 & 48 & & \\
\hline $3 s 4 p^{3} P_{2}^{\circ}-3 s 4 d^{3} D_{3}$ & 380.654 & 18.8 & $78.4 \pm 14.6$ & 118 & 69 & 47 & & \\
\hline $3 \mathrm{~s} 4 \mathrm{f}^{1} \mathrm{~F}_{3}^{\circ}-3 \mathrm{~s} 5 \mathrm{~g}{ }^{1} \mathrm{G}_{4}$ & 392.447 & 19.0 & $84.6 \pm 16.9$ & 163 & & & & \\
\hline $3 \mathrm{p}^{2}{ }^{1} \mathrm{~S}_{0}-3 \mathrm{~s} 4 \mathrm{p}{ }^{1} \mathrm{P}_{1}^{\circ}$ & 433.850 & 19.0 & $26.9 \pm 5.3$ & & & & & \\
\hline $3 s 4 s^{3} S_{1}-3 s 4 p{ }^{3} P_{2}^{\circ}$ & 455.262 & 19.0 & $51.2 \pm 9.2$ & & & & 18 & 48 \\
\hline $3 s 4 s^{3} S_{1}-3 s 4 p{ }^{3} P_{1}^{\circ}$ & 456.782 & 19.0 & $54.2 \pm 9.8$ & & & & 18 & 56 \\
\hline $3 s 4 s^{3} S_{1}-3 s 4 p{ }^{3} P_{0}^{\circ}$ & 457.476 & 19.0 & $48.5 \pm 9.3$ & & & 306 & 18 & \\
\hline $3 \mathrm{~s} 4 \mathrm{~d}^{1} \mathrm{D}_{2}-3 \mathrm{~s} 5 \mathrm{f}{ }^{1} \mathrm{~F}_{3}^{\circ}$ & 471.665 & 19.0 & $181 \pm 36$ & 277 & & & & \\
\hline $3 \mathrm{~s} 4 \mathrm{f}^{3} \mathrm{~F}_{2}^{\circ}-3 \mathrm{~s} 5 \mathrm{~g}^{3} \mathrm{G}_{3}$ & 481.333 & 19.0 & $398 \pm 79$ & 438 & 398 & & & \\
\hline $3 \mathrm{~s} 4 \mathrm{f}^{3} \mathrm{~F}_{3}^{\circ}-3 \mathrm{~s} 5 \mathrm{~g}^{3} \mathrm{G}_{4}$ & 481.972 & 19.0 & $423 \pm 84$ & 438 & 425 & & & \\
\hline $3 \mathrm{~s} 4 \mathrm{f}^{3} \mathrm{~F}_{4}^{\circ}-3 \mathrm{~s} 5 \mathrm{~g}^{3} \mathrm{G}_{5}$ & 482.897 & 19.0 & $421 \pm 84$ & 438 & 405 & & & \\
\hline $3 \mathrm{~s} 4 \mathrm{~s}{ }^{1} \mathrm{~S}_{0}-3 \mathrm{~s} 4 \mathrm{p}{ }^{1} \mathrm{P}_{1}^{\circ}$ & 573.973 & 19.0 & $98.2 \pm 20.0$ & & 87 & & & \\
\hline
\end{tabular}

Note 4. Our measured Si III Stark FWHM ( $W_{\mathrm{m}}$ in pm) at given $T$ (in $10^{3} \mathrm{~K} \pm 14 \%$ ) and $N=1 \times 10^{23} \mathrm{~m}^{-3} \pm 13 \%$, with estimated accuracies. The $W$ (in pm) values from other experiments are also given: $\mathrm{m}$ (Djeniže et al. 1992), $\mathrm{n}$ (Gonzalez et al. 2000), p (Kusch \& Schröder 1982), q (Platiša et al. 1977), and $\mathrm{r}$ (Purić et al. 1974) obtained at following $T$ and $N: \mathrm{m}\left(T=4800 \mathrm{~K}, N=2.6 \times 10^{23} \mathrm{~m}^{-3}\right), \mathrm{n}\left(T=19000 \mathrm{~K}, N=1.0 \times 10^{23} \mathrm{~m}^{-3}\right)$, $\mathrm{p}\left(T=23500 \mathrm{~K}, N=1.0 \times 10^{23} \mathrm{~m}^{-3}\right), \mathrm{q}\left(T=25600 \mathrm{~K}, N=5.8 \times 10^{22} \mathrm{~m}^{-3}\right)$, and r $\left(T=8700 \mathrm{~K}, N=1.0 \times 10^{23} \mathrm{~m}^{-3}\right)$. Atomic data are taken from NIST. 


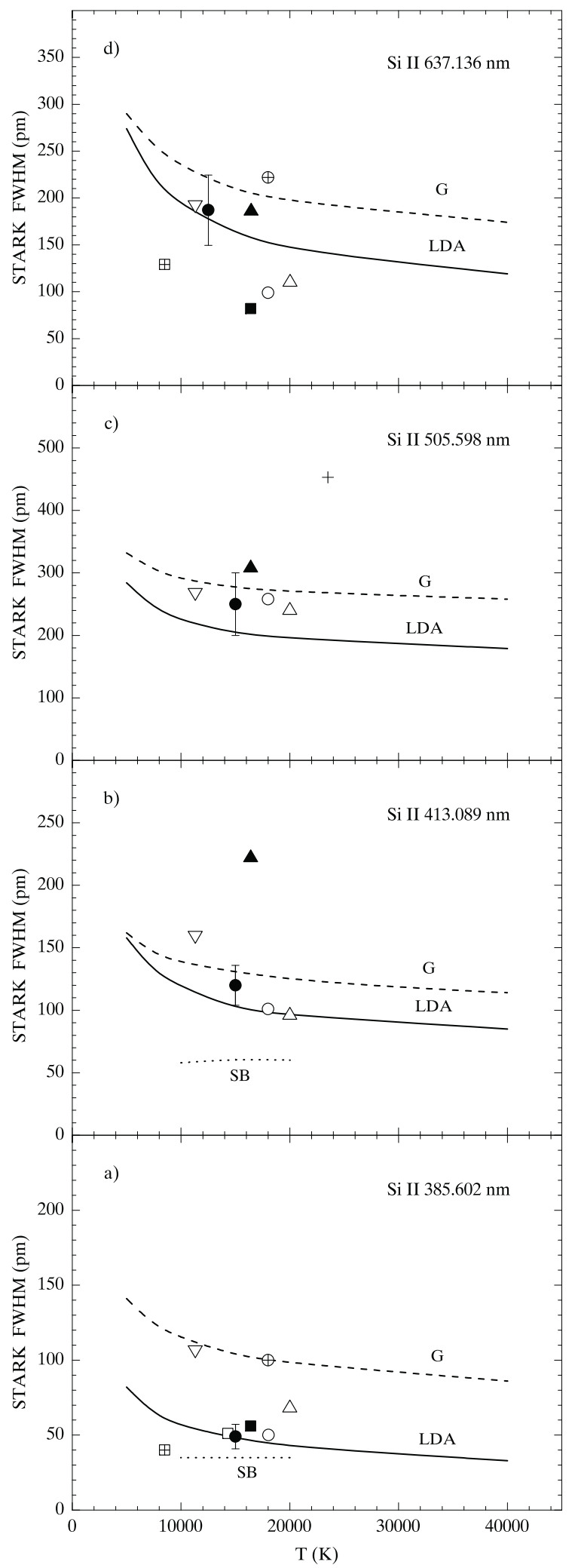

Fig. 10. Panels a-d). The measured and calculated Stark $F W H M$ (in pm) versus electron temperature $(T$ in $\mathrm{K})$ at $1 \times 10^{23} \mathrm{~m}^{-3}$ electron density for the most investigated Si II line profiles. The symbols mean: - this work; ○ (Gonzalez et al. 2002); $\square$ (Wollschläger et al. 1997); ム (Peréz et al. 1993); $\Delta$ (Lesage et al. 1983); + (Kusch \& Schröder 1982); $\oplus$ (Chiang \& Griem 1978); $\nabla$ (Lesage et al. 1977); (Purić et al. 1974), and $\otimes$ (Konjević et al. 1970). The SB, G and LDA denote values calculated by: Sahal-Bréchot (1968), Griem (1974), and Lanz et al. (1988) only for the electrons as perturbers. Error bars represent estimated accuracies in this work (see Table 3 ).
Table 5. The measured Si IV Stark widths.

\begin{tabular}{cccccc}
\hline \hline Transition & $\lambda(\mathrm{nm})$ & $T$ & $W_{\mathrm{m}}$ & $\mathrm{t}$ & $\mathrm{u}$ \\
\hline $4 \mathrm{p}{ }^{2} \mathrm{P}_{1 / 2}^{\circ}-5 \mathrm{~s}^{2} \mathrm{~S}_{1 / 2}$ & 212.018 & 18.8 & $19.5 \pm 3.9$ & 19 & \\
$4 \mathrm{p}{ }^{2} \mathrm{P}_{3 / 2}^{\circ}-5 \mathrm{~s}^{2} \mathrm{~S}_{1 / 2}$ & 212.747 & 18.8 & $22.2 \pm 4.4$ & 19 & \\
$4 \mathrm{p}^{2} \mathrm{P}_{1 / 2}^{\circ}-4 \mathrm{~d}^{2} \mathrm{D}_{3 / 2}$ & 314.956 & 19.0 & $28.7 \pm 3.2$ & $66^{*}$ & 14 \\
$4 \mathrm{p}^{2} \mathrm{P}_{3 / 2}^{\circ}-4 \mathrm{~d}^{2} \mathrm{D}_{5 / 2}$ & 316.571 & 19.0 & $29.6 \pm 3.0$ & 34 & 14 \\
$4 \mathrm{~d}^{2} \mathrm{D}_{3 / 2}-5 \mathrm{p}{ }^{2} \mathrm{P}_{3 / 2}^{\circ}$ & 376.244 & 18.8 & $66.5 \pm 13.3$ & 58 & \\
$4 \mathrm{~d}^{2} \mathrm{D}_{3 / 2}-5 \mathrm{p}^{2} \mathrm{P}_{1 / 2}^{\circ}$ & 377.315 & 18.8 & $56.2 \pm 11.2$ & 46 & \\
$4{ }^{2} \mathrm{~S}_{1 / 2}-4 \mathrm{p}^{2} \mathrm{P}_{3 / 2}^{\circ}$ & 408.885 & 19.0 & $29.6 \pm 5.3$ & 44 & 12 \\
$4 \mathrm{~s}^{2} \mathrm{~S}_{1 / 2}-4 \mathrm{p}{ }^{2} \mathrm{P}_{1 / 2}^{\circ}$ & 411.610 & 19.0 & $26.5 \pm 4.8$ & & 12 \\
The transition is not given & 251.751 & 19.0 & $35.1 \pm 7.0$ & & \\
\hline
\end{tabular}

Note 5. Our measured Si IV Stark FWHM $\left(W_{\mathrm{m}}\right.$ in pm) at given $T$ (in $10^{3} \mathrm{~K} \pm 14 \%$ ) and $N=1 \times 10^{23} \mathrm{~m}^{-3} \pm 13 \%$, with estimated accuracies. The $W$ (in pm) values from other experiments are also given: $\mathrm{t}$ (Djeniže et al. 1992) and u (Platiša et al. 1977) obtained at following $T$ and $N$ : $\mathrm{t}\left(T=29000 \mathrm{~K}, N=1.6 \times 10^{23} \mathrm{~m}^{-3}\right)$ and $\mathrm{u}(T=25600 \mathrm{~K}, N=5.8 \times$ $\left.10^{22} \mathrm{~m}^{-3}\right)$. Asterisk denotes value measured by Djeniže et al. (1992) at $T=48000 \mathrm{~K}$ and $N=2.6 \times 10^{23} \mathrm{~m}^{-3}$. Atomic data are taken from NIST.

and $212.747 \mathrm{~nm}$ ), though, agreement is encouraging - better than $\pm 5 \%$. We would like to emphasize that existing experimental Si IV $W$ values are also lower than the calculated by Dimitrijević et al. (1991).

The Si III and Si IV spectra were recorded later, around the 30th $\mu$ s from the beginning of the discharge. We have found that at this stage of the discharge Si III and Si IV spectral line profiles are well defined, while the continuum is inconsiderable (see Figs. 3, 4, and 6).

\section{Conclusions}

The neutral and ionized silicon spectral line shapes were investigated in the laboratory helium plasma at electron densities ranging between $3.7 \times 10^{22} \mathrm{~m}^{-3}$ and $1.1 \times 10^{23} \mathrm{~m}^{-3}$ and electron temperatures between $12500 \mathrm{~K}$ and $19000 \mathrm{~K}$. The spectral line profiles were recorded using a highly sensitive spectrograph (McPherson) and ICCD camera (Andor) detection system.

The Stark FWHMs of $13 \mathrm{Si} \mathrm{I}, 15 \mathrm{Si}$ II, $28 \mathrm{Si}$ III, and $9 \mathrm{Si}$ IV spectral lines were measured in the wavelength interval between $206 \mathrm{~nm}$ and $640 \mathrm{~nm}$. Five Si I, four Si II, eleven Si III values and one Si IV $W$ value from the investigated set have not been measured or calculated so far. Our $W$ values are compared with the existing theoretical and experimental data.

At the mentioned plasma parameters tolerable agreement was found (within the accuracy of the experiment and uncertainties of the theoretical approaches used) among our measured and calculated Stark FWHM values. We recommend the Stark FWHMs of the intense $254.182 \mathrm{~nm}, 308.624 \mathrm{~nm}$ and $309.342 \mathrm{~nm}$ Si III, and the $314.956 \mathrm{~nm}$ and $316.571 \mathrm{~nm}$ Si IV lines for purpose of plasma diagnostics.

Acknowledgements. This work is part of the "Determination of atomic parameters on the basis of spectral line profiles" (ON141010) project supported by the Ministry of Science and Technological Development of the Republic of Serbia.

\section{Appendix A}

It is interesting to estimate the maximum amount of selfabsorption, which, if present, would still be consistent with our data. Several procedures for correcting spectral line profiles due to self-absorption are in use (Konjevic 1999; Escarguel et al. 2000). The fact that six Si I resonant spectral lines, considered 
in this paper, effectively have the same lower level gives us possibility for a specific analysis of the presented data.

It is well known that for homogenous plasma layer of length $l$ intensity of the emitted radiation per unit wavelength (the radiance) is (Hilborn 1982):

$I_{\lambda}=F_{\lambda}\left[1-\exp \left(-k_{\lambda} l\right)\right]$

where $F_{\lambda}$ denotes the radiance per unit length per unit wavelength, while $k_{\lambda}$ is the absorption coefficient at the wavelength $\lambda$. Product $k_{\lambda} l$ is the optical depth - the quantity which determines the amount of self-absorption. For very weak self-absorption, $k_{\lambda} l \ll 1$, after preserving only the first two terms in the Maclaurin expansion of the exponential function $\exp \left(k_{\lambda} l\right) \sim$ $1-k_{\lambda} l$, the radiance of homogenous plasma layer simplifies to $I_{\lambda}=F_{\lambda} l \equiv I_{0 \lambda}$. Suppose that self-absorption is slightly higher, so that we need first three terms in the expansion of the exponential function. Equation (1) becomes

$I_{\lambda}=I_{0 \lambda}\left[1-k_{\lambda} l / 2\right]$.

Coefficient $k_{\lambda}$ depends on oscillator strength $f_{12}$ and density of the lower level of the transition $n$, i.e. $k_{\lambda}=\frac{e^{2} \lambda^{2}}{4 \epsilon_{0} m c^{2}} g_{1} f_{12} n z(\lambda)$, where $e$ is the electron charge, $\epsilon_{0}$ the vacuum permittivity, $c$ the speed of light, $m$ the electron mass, $g_{1}$ the statistical weight of the lower level of the transition and $z(\lambda)$ the normalized profile of the spectral line, $\int_{-\infty}^{\infty} z(\lambda) \mathrm{d} \lambda=1$. Inserting $k_{\lambda}$ into Eq. (2) and integrating over the line profile we have

$I=I_{0}\left[1-\frac{e^{2} \lambda^{2} l}{8 \epsilon_{0} m c^{2}} g_{1} f_{12} n \int_{-\infty}^{\infty} z^{2}(\lambda) \mathrm{d} \lambda\right]$.

In Eq. (3) $I$ is the reduced spectral line intensity due to selfabsorption, while $I_{0}$ is the one that would be obtained in a case of negligible self-absorption. We introduce quantity $Z^{2}=$ $\int_{-\infty}^{\infty} z^{2}(\lambda) \mathrm{d} \lambda$. For common line profiles $Z^{2}=(C \cdot W)^{-1}$ where $W$ is the halfwidth of the line, $C$ is the dimensionless constant equal to $2 \pi$ for Lorentz profile and $\sqrt{2 \pi / \ln 2}$ for Gauss. Usually $z(\lambda)$ is not known, but for a weak self absorbtion, one can take the experimental value for $W$ sacrifiing a few percent in exactness. If we have two measured intensities, for spectral lines $p$ and $q$ with the same lower level, their ratio will be

$\frac{I_{p}}{I_{q}}=\frac{I_{0 p}}{I_{0 q}}\left[\frac{1-\frac{e^{2} \lambda_{p}^{2} l}{8 \epsilon_{0} m c^{2}} g_{p} f_{p} n Z_{p}^{2}}{1-\frac{e^{2} \lambda_{q}^{2} l}{8 \epsilon_{0} m c^{2}} g_{q} f_{q} n Z_{q}^{2}}\right]$.

Here we applied a simplified notation for oscillator strengths $f_{p} \equiv f_{12}^{p}$ and $f_{q} \equiv f_{12}^{q}$, in a similar way $g_{p} \equiv g_{1}^{p}$ and $g_{q} \equiv g_{1}^{q}$. For lines from the same multiplet, $\frac{I_{0 p}}{I_{0 q}}=\frac{A_{p} g_{p} \lambda_{q}}{A_{q} g_{q} \lambda_{p}}$, where $A_{p}, A_{q}$ are transition probabilities for the lines $p$ and $q$. From Eq. (4) one can express density of the lower level of the transition, $n$, as

$n=\frac{Q_{p, q}-1}{\left(Q_{p, q} \frac{1}{W_{q}} g_{q} f_{q} \lambda_{q}^{2}-\frac{1}{W_{p}} g_{p} f_{p} \lambda_{p}^{2}\right)} \frac{8 C \epsilon_{0} m c^{2}}{e^{2} l}$.

Quantity $Q_{p, q}=\frac{I_{p}}{I_{q}} \frac{A_{q} g_{q} \lambda_{p}}{A_{p} g_{p} \lambda_{q}}$ is already defined in Sect. 4.2 and tabulated in Table 1. Taking into account that, for lines from the same multiplet $\lambda_{1} \sim \lambda_{2} \sim \lambda_{3} \cdots=\lambda$, Eq. (5) simplifies to

$n=\frac{Q_{p, q}-1}{\left(Q_{p, q} \frac{1}{w_{q}} g_{q} f_{q}-\frac{1}{w_{p}} g_{p} f_{p}\right)} \frac{8 C W_{0} \epsilon_{0} m c^{2}}{e^{2} l \lambda^{2}}$,

where $w_{p}, w_{q}$ are dimensionless numbers expressing walfwidths of the lines $p$ and $q$ in picometers, i.e. $W_{i}=w_{i} \cdot W_{0}$ where $i=\{p, q\}$ and $W_{0}=1 \times 10^{-12} \mathrm{~m}$. There are $N=6$ independent values of quotient $Q$ for the six Si I resonant lines ${ }^{2}$ given in Table 1, which will result in 6 values for $n$. Ideally, all values should be the same. Due to uncertainties of measured intensities $I$ and halfwidths of the line profiles $W$ and uncertainties of tabulated $g f$ data one will get a set of 6 different values for $n$. We can easily calculate the mean value $\bar{n}=\frac{\sum_{i} n_{i}}{N}$ and the error of the mean value $\Delta n=\sqrt{\frac{\sum_{i}\left(\bar{n}-n_{i}\right)^{2}}{N(N-2)}}$ of the set. In practice, one should remove obvious outliers from the data set before calculating $\bar{n}, \Delta n$. For the data presented in Table 1 and adopting ${ }^{3} C=4$, we found $\bar{n}=(0.95 \pm 0.83) \times 10^{17} \mathrm{~m}^{-3}$. This result allows calculation of optical depth for each spectral line $\quad k_{\lambda} l=\frac{e^{2} \lambda^{2} l}{4 \epsilon_{0} m c^{2}} g_{1} f_{12} \bar{n} z(\lambda)$. Obviously, the amount of the self-absorption for a particular line is proportional to the product $g f$. It is common practice to apply adequate analytical or numerical procedure to correct spectral line profile if the optical depth $k_{\lambda} l<1$. For the Si I resonant spectral lines optical depth, at our discharge conditions, is in the range $0.07<k_{\lambda} l<0.38$. Therefore, by relying on Eq. (2) one can correct measured radiance $I_{\lambda}$ of the spectral line at the particular wavelength $\lambda$ to be close to the value $I_{0 \lambda}$ which would be obtained in the case of the negligible self-absorption

$I_{o \lambda}=\frac{I_{\lambda}}{1-\frac{k_{\lambda} l}{2}}$.

Relying on Eq. (7) we evaluated corrections of the Stark widths given in Table 2 .

\section{References}

Arsenijević, V., Fabbro, S., Mourao, A. M., \& Rica da Silva, A. J. 2008, A\&A, 492, 535

Bongard, S., Baron, E., Smadja, G., Branch, D., \& Hauschildt, P. H. 2008, ApJ, 687,456

Bukvić, S., \& Spasojević, D. 2005, Spectrochim. Acta B, Atomic Spectr., 60, 1308

Bukvić, S., Spasojević, D., \& Žigman, V. 2008, A\&A, 477, 967

Catanzaro, G., \& Leone, F. 2008, MNRAS, 389, 1414

Catanzaro, G., Leone, F., Busa, I., \& Romano, P. 2008, New Astron., 13, 113

Chiang, T., \& Griem, H. R. 1978, Phys. Rev. A, 18, 1169

Danforth, C. W., \& Shull, M. J. 2008, ApJ, 679, 194

Davies, J. T., \& Vaughan, J. M. 1963, ApJ, 137, 1302

Dimitrijević, M. S., \& Konjević, N. 1981, Spectral Line Shapes, ed. B. Wende (Berlin: W. de Gruyter)

Dimitrijević, M. S., Sahal-Bréchot, S., \& Bommier, V. 1991, A\&ASS, 89, 591

Dimitrijević, M. S., Ryabchikova, T., Simić, Z., Popović, L. Č., \& Daćić, M. 2007, A\&A, 469, 681

Djeniže, S. 2009, Spectrochim. Acta B, Atomic Spectr., 64, 242

Djeniže, S., \& Bukvić, S. 2001, A\&A, 365, 252

Djeniže, S., Srećković, A., Labat, J., Purić, J., \& Platiša, M. 1992, J. Phys. B, 25, 785

Djeniže, S., Dimitrijević, M. S., Srećković, A., \& Bukvić, S. 2002, A\&A, 396, 331

Drawin, H. W., \& Felenbok, P. 1965, Data for Plasmas in Local Thermodynamic Equilibrium (Paris: Gauthier-Villars)

Escarguel, A., Ferhat, B., Lesage, A., \& Richou, J. 2000, JQSRT, 64, 353

Fox, A. J., Ledoux, C., Vreeswijk, P. M., Smette, A., \& Jaunsen, A. O. 2008, A\&A, 491, 189

Gomez de Castro, A. I., \& Verdugo, E. 2007, ApJ, 654, L91

Gonzalez, V. R., Aparicio, J. A., del-Val, A., \& Mar, S. 2000, A\&A, 363, 1177

Gonzalez, V. R., Aparicio, J. A., del-Val, A., \& Mar, S. 2002, J. Phys. B: At. Mol. Opt. Phys., 35, 3557

2 In general for $N$ spectral lines there are $N ! /(2(N-2)$ !) independent combinations; however, we have two groups of three spectral lines measured at two different temperatures. We suppose that the density of the effective lower level of the multiplet is the same for both temperatures. 3 Constant $C$ for Voigt profile is less than $2 \pi$ (Lorentz) and above $\sqrt{2 \pi / \ln 2}$ (Gauss), and it has to be evaluated separately for a specific Voigt profile. 
Griem, H. R. 1964, Plasma Spectroscopy (New York: McGraw Hill)

Griem, H. R. 1974, Spectral Line Broadening by Plasmas (New York: Acad. Press)

Hamann, F., Kaplan, K. F., Hidalgo, R. P., Prochaska, J. X., \& Herbert-Fort, S. 2008, MNRAS, 391, L39

Hilborn, R. C. 1982, Am. J. Phys., 50, 982

Hutchinson, I. H. 2002, Principles of plasma diagnostics (Cambridge University Press), second edition

Iijima, T., \& Nakanishi, H. 2008, A\&A, 482, 865

Kawka, A., Vennes, S., Dupuis, J., Chayer, P., \& Lanz, T. 2008, ApJ, 675, 1518

Konjević, N. 1999, Phys. Rep., 316, 339

Konjević, N., Purić, J., Ćirković, Lj., \& Labat, J. 1970, J. Phys. B, 3, 999

Konjević, N., Lesage, A., Fuhr, J. R., \& Wiese, W. L. 2002, J. Phys. Chem. Ref. Data, 31, 819

Kruithof, A. A., \& Penning, F. M. 1937, Physica, 4, 430

Kusch, H. J., \& Schröder, K. 1982, A\&A, 116, 255

Lanz, T., Dimitrijević, M. S., \& Artru, M.-C. 1988, A\&A, 192, 249

Lesage, A. 1994, Astrophysical Applications of Powerful New Databases, ed.

S. J. Adelman, \& W. L. Wiese, Astron. Soc. Pacific Conf. Ser., 78

Lesage, A. 2009, New Astron. Rev., 52, 471

Lesage, A., \& Redon, R. 2004, A\&A, 418, 765

Lesage, A., Sahal-Bréchot, S., \& Miller, M. H. 1977, Phys. Rev. A, 16, 1617
Lesage, A., Rathore, B. A., Lakićević, I. S., \& Purić, J. 1983, Phys. Rev. A, 28, 2264

Meyer, J., \& Beck, R. J. 1970, A\&A, 8, 93

Miller, M. H., \& Bengtson, R. D. 1970, Phys. Rev. A, 1, 983

Milosavljević, V., \& Djeniže, S. 2001, Eur. Phys. J. D, 15, 99

Nehme, C., Gry, C., Boulanger, F., et al. 2008, A\&A, 483, 471

NIST 2009, Atomic Spectra Database Lines (wavelength order), http://www . physics.nist.gov

Peréz, C., de la Rosa, I., de Frutos, A. M., \& Mar, S. 1993, Phys. Rev. E, 47, 756

Platiša, M., Dimitrijević, M. S., Popović, M., \& Konjević, N. 1977, J. Phys. B, 10,2997

Purić, J., Djeniže, S., Labat, J., \& Ćirković, Lj. 1974, Z. Phys., 267, 71

Rogowski, W., \& Steinhaus, W. 1912, Archiv für Elektrotechnik, 1, 141

Rompe, R., \& Stenbeeck, M. 1967, Ergebnisse der Plasmaphysik und der Gaselektronik (Berlin: Akademie Verlag)

Sahal-Bréchot, S. 1968, Z. Astrophys., 69, 74

Shi, J. R., Gehren, T., Butler, K., Mashonkina, L. I., \& Zhao, G. 2008, A\&A, 486, 303

Silfvast, W. T. 1971, Phys. Rev. Lett., 27, 1489

Srećković, A., Bukvić, S., \& Djeniže, S. 1998, Phys. Scr., 57, 225

Sundqvist, J. O., Ryde, N., Harper, G. M., Kruger, A., \& Richter, M. J. 2008, A\&A, 486, 985

Wollschläger, F., Mitsching, J., Meiners, D., et al. 1997, JQSRT, 58, 135 